\title{
Deployment of convalescent plasma for the prevention and treatment of COVID-19
}

\author{
Evan M. Bloch, ${ }^{1}$ Shmuel Shoham, ${ }^{2}$ Arturo Casadevall, ${ }^{3}$ Bruce S. Sachais, ${ }^{4}$ Beth Shaz,${ }^{4}$ Jeffrey L. Winters, ${ }^{5}$ Camille van Buskirk, ${ }^{5}$ \\ Brenda J. Grossman, ${ }^{6}$ Michael Joyner, Jeffrey P. Henderson, ${ }^{8,9}$ Andrew Pekosz, ${ }^{3}$ Bryan Lau, ${ }^{10}$ Amy Wesolowski, ${ }^{10}$ Louis Katz, ${ }^{11}$ \\ Hua Shan, ${ }^{12}$ Paul G. Auwaerter, ${ }^{2}$ David Thomas, ${ }^{2}$ David J. Sullivan, ${ }^{3}$ Nigel Paneth, ${ }^{13,14}$ Eric Gehrie, ${ }^{1}$ Steven Spitalnik, ${ }^{15}$ \\ Eldad A. Hod, ${ }^{15}$ Lewis Pollack, ${ }^{16}$ Wayne T. Nicholson, ${ }^{7}$ Liise-anne Pirofski, ${ }^{17}$ Jeffrey A. Bailey, ${ }^{18}$ and Aaron A.R. Tobian ${ }^{1}$ \\ ${ }^{1}$ Division of Transfusion Medicine, Department of Pathology, ${ }^{2}$ Department of Infectious Diseases, School of Medicine, and ${ }^{3}$ Department of Molecular Microbiology and Immunology, Bloomberg School of \\ Public Health, Johns Hopkins University, Baltimore, Maryland, USA. ${ }^{4}$ New York Blood Center Enterprises, New York, New York, USA. ${ }^{5}$ Division of Transfusion Medicine, Department of Laboratory Medicine \\ and Pathology, Mayo Clinic, Rochester, Minnesota, USA. 'Department of Pathology, Washington University School of Medicine in St. Louis, St. Louis, Missouri, USA. ${ }^{7}$ Department of Anesthesiology and \\ Perioperative Medicine, Mayo Clinic, Rochester, Minnesota, USA. ${ }^{8}$ Department of Medicine and ${ }^{9}$ Department of Molecular Microbiology, Washington University School of Medicine in St. Louis, St. Louis, \\ Missouri, USA. ${ }^{10}$ Department of Epidemiology, Bloomberg School of Public Health, Johns Hopkins University, Baltimore, Maryland, USA. "'Mississippi Valley Regional Blood Center, Davenport, lowa, USA \\ ${ }^{12}$ Department of Pathology, Stanford University School of Medicine, Palo Alto, California, USA. ${ }^{13}$ Department of Epidemiology and Biostatistics and ${ }^{14}$ Department of Pediatrics and Human Development, \\ Michigan State University, East Lansing, Michigan, USA. ${ }^{15}$ Department of Pathology and Cell Biology, Columbia University Irving Medical Center, New York-Presbyterian Hospital, New York, New York, USA. \\ ${ }^{16}$ Independent consultant, Princeton, New Jersey. ${ }^{17}$ Division of Infectious Diseases, Albert Einstein College of Medicine and Montefiore Medical Center, New York, New York, USA. ${ }^{18}$ Department of Pathology \\ and Laboratory Medicine, Warren Alpert Medical School, Brown University, Providence, Rhode Island, USA.
}

Severe acute respiratory syndrome coronavirus 2 (SARS-CoV-2), the cause of coronavirus disease 2019 (COVID-19), has spurred a global health crisis. To date, there are no proven options for prophylaxis for those who have been exposed to SARS-CoV-2, nor therapy for those who develop COVID-19. Immune (i.e., "convalescent") plasma refers to plasma that is collected from individuals following resolution of infection and development of antibodies. Passive antibody administration through transfusion of convalescent plasma may offer the only short-term strategy for conferring immediate immunity to susceptible individuals. There are numerous examples in which convalescent plasma has been used successfully as postexposure prophylaxis and/or treatment of infectious diseases, including other outbreaks of coronaviruses (e.g., SARS-1, Middle East respiratory syndrome [MERS]). Convalescent plasma has also been used in the COVID-19 pandemic; limited data from China suggest clinical benefit, including radiological resolution, reduction in viral loads, and improved survival. Globally, blood centers have robust infrastructure for undertaking collections and constructing inventories of convalescent plasma to meet the growing demand. Nonetheless, there are nuanced challenges, both regulatory and logistical, spanning donor eligibility, donor recruitment, collections, and transfusion itself. Data from rigorously controlled clinical trials of convalescent plasma are also few, underscoring the need to evaluate its use objectively for a range of indications (e.g., prevention vs. treatment) and patient populations (e.g., age, comorbid disease). We provide an overview of convalescent plasma, including evidence of benefit, regulatory considerations, logistical work flow, and proposed clinical trials, as scaleup is brought underway to mobilize this critical resource.

\section{Introduction}

Severe acute respiratory syndrome coronavirus 2 (SARS-CoV-2), the cause of coronavirus disease 2019 (COVID-19), has been declared a pandemic (1). As of the time of this writing, over 1.2 million cases of COVID-19 have been reported, spanning 181 countries or regions and contributing to over 64,000 deaths (2). Despite a global health crisis that is unparalleled in modern history, there are currently no proven options for prophylaxis for those who have been exposed to SARSCoV-2 nor is there therapy for those who go on to develop COVID-19.

Immune (i.e. "convalescent") plasma refers to plasma that is collected from individuals following resolution of infection and

Conflict of interest: EMB is a member of the FDA Blood Products Advisory Committee. Copyright: () 2020, American Society for Clinical Investigation.

Reference information: J Clin Invest. 2020;130(6):2757-2765.

https://doi.org/10.1172/JCl138745. development of antibodies. Passive antibody therapy, through transfusion of convalescent plasma, may prevent clinical infection or blunt clinical severity in individuals with recent pathogen exposure. Antibody therapy can also be used to treat patients who are already manifesting symptoms of varying severity. However, passive antibody therapy is most effective when administered prophylactically or used early after the onset of symptoms $(3,4)$.

Passive antibody therapy has been in use for over a century (5). The active agents are antibodies against the target pathogen of interest. Today, passive antibody therapy relies primarily on pooled immunoglobulin preparations that contain high concentrations of antibodies. In contrast, plasma has been used emergently in epidemics where there is insufficient time or resources to generate immunoglobulin preparations. There are multiple examples, both historical and recent, in which convalescent plasma was employed successfully as postexposure prophylaxis (e.g., hepatitis, mumps, 
polio, measles, rabies) and/or treatment for a myriad of infectious diseases (e.g., influenza, Argentine hemorrhagic fever, SARS$\mathrm{CoV}$, Middle East respiratory syndrome [MERS], Ebola), favorably affecting a range of laboratory (e.g., viral loads, cytokine responses) and clinical outcomes (notably mortality) (6-12).

\section{Mechanism of action}

The antibodies present in immune (i.e., convalescent") plasma mediate their therapeutic effect through a variety of mechanisms. An antibody can bind to a given pathogen (e.g., virus), thereby neutralizing its infectivity directly, while other antibody-mediated pathways, such as complement activation, antibody-dependent cellular cytotoxicity, and/or phagocytosis, may also contribute to its therapeutic effect. Nonneutralizing antibodies that bind to the pathogen - but do not interfere with its ability to replicate in in vitro systems - may also contribute to prophylaxis and/or enhance recovery $(13,14)$. Importantly, passive antibody administration offers the only short-term strategy for conferring immediate immunity to susceptible individuals. This is particularly the case in the setting of a novel, emerging infectious disease such as SARS-CoV-2/COVID-19. While fractionated plasma products (e.g., hyperimmune globulin, mAbs) and/or vaccination may offer durable therapeutic options, human anti-SARS-CoV-2 plasma is the only therapeutic strategy that is immediately available for use to prevent and treat COVID-19.

\section{The use of convalescent plasma against coronaviruses}

Convalescent plasma has been used in two other coronavirus epidemics in the 21st century: SARS1 in 2003 and MERS from 2012 to the present. Experience from those outbreaks shows that convalescent plasma contains neutralizing antibodies (15). The largest study involved the treatment of 80 patients in Hong Kong with SARS1 (16). Compared with those given plasma later, patients who were treated before day 14 had improved outcomes, as defined by discharge from hospital before day 22, supporting early administration for optimal effect. Limited data also suggested benefit in seriously ill individuals: three patients with SARS-CoV-1 infection in Taiwan were treated with convalescent plasma, resulting in a reduction in viral load; all three recipients survived (17). Treatment with convalescent plasma was also reported in three patients in South Korea with MERS (18). Treatment using convalescent plasma in patients with MERS was limited by a small pool of donors with sufficient antibody levels (19). Reported dosages and characterization of convalescent plasma (i.e., with respect to antibody titers) is highly variable (Table 1 ).

In this current pandemic, there are reports that convalescent plasma has been used in China to treat patients with COVID-19 $(20,21)$. In a pilot study of 10 patients with severe COVID-19, the investigators collected convalescent plasma with neutralizing antibody titers at or exceeding a 1:640 dilution (22). Transfusion of convalescent plasma resulted in no serious adverse effects in the recipients. All 10 patients had improvement in symptoms (e.g., fever, cough, shortness of breath, and chest pain) within 1 to 3 days of transfusion; they also demonstrated radiological improvement in pulmonary lesions. In seven RNA-emic patients, transfusion of convalescent plasma was temporally associated with undetect- able viral loads. Further, screening of 39 of 40 (97.5\%) of recovered COVID-19 patients displayed neutralizing antibody titers for 160 or more. A case series of five critically ill patents in China also reported improvement in clinical status following transfusion with convalescent plasma (SARS-CoV-2 IgG titers >1000), as evidenced by weaning off mechanical ventilation, reduction in viral loads, improved oxygenation, and clinical stabilization (21). Although constrained by small sample sizes and limitations of study design and concomitant treatment modalities (e.g., remdesivir, ribavirin, corticosteroids, etc.), these findings suggest that administration of convalescent plasma is safe, reduces viral load, and may improve clinical outcomes. This has led to calls for the wider adoption of convalescent plasma for COVID-19 (23). Nonetheless, while the data support safety and potential efficacy of convalescent plasma, randomized trials are needed (23). Similarly, high-dose intravenous immunoglobulin (IVIg) has been suggested as a potential therapy for COVID-19 (24); however, supporting data are few and marred by potential confounders.

\section{Regulatory oversight and access to convalescent plasma}

On March 24, 2020, the US FDA published its guidance document for investigational COVID-19 convalescent plasma (25). The document outlines three pathways for access to convalescent plasma. The first is under an emergency use investigational new drug (IND) application. This allows a provider to apply for compassionate use in an individual patient with severe or immediately life-threatening COVID-19. Of note, this guidance document does not allow for prophylaxis. Minimal requirements (e.g., a brief history and description of indication) enable those requests to be expedited. The second is a traditional pathway to apply for an IND to support research (e.g., for clinical trials). Finally, a government-led initiative provides expanded access of convalescent plasma to participating institutions under a master treatment protocol. The latter approach enables the collection of extensive data, albeit through a nonrandomized study design.

\section{Convalescent plasma collections workflow}

Convalescent plasma can be mobilized rapidly using the established blood collection and transfusion infrastructure. Specifically, convalescent plasma is obtained and administered using standard collection and transfusion practices that are available around the world. As the number of individuals who resolve their infections increases, so does the number of potential eligible donors of convalescent plasma. Nonetheless, there are multiple logistical hurdles if we are to procure a satisfactory inventory of convalescent plasma. As depicted in Figure 1, a workflow has been developed to illustrate the individual steps that need to be undertaken spanning assessment of donor eligibility, donor recruitment, collections, and transfusion itself. Each brings its own challenges.

Donor eligibility. First is the question of what constitutes a convalescent donor. Relying only on absence of symptoms invites test-seeking behavior that could overwhelm - or at least burden unnecessarily - collection services with inappropriate donors. The criteria for individuals to be eligible to donate convalescent plasma include a history of COVID-19, either as confirmed by approved molecular testing (e.g., nasopharyngeal [NP] swab) or 
Table 1. Dosing of convalescent plasma in coronavirus epidemics

\begin{tabular}{|c|c|c|c|c|c|}
\hline Disease & Location & Dose of CP & Titer & Summary finding & Reference \\
\hline SARS1 & Hong Kong, China & $\begin{array}{l}\text { Mean volume } 279.3 \pm \\
127.1 \mathrm{~mL}(\text { range, } \\
160-640 \mathrm{~mL} \text { ) }\end{array}$ & Not performed & $\begin{array}{l}\text { - Retrospective chart review of } 80 \text { patients who received CP } \\
\text { - 14 (range, 7-30 days) following the onset of symptoms } \\
\text { - Cood clinical outcome in } 33 \text { ( } 41.3 \% \text { ) patients as defined by } \\
\text { hospital discharge by day } 22 \\
\text { - Improved outcome associated with early administration } \\
\text { - No adverse events }\end{array}$ & 16 \\
\hline SARS1 & Taipei, Taiwan & $500 \mathrm{~mL}$ & Serum antibody (IgG) titer was $>640$ & $\begin{array}{l}\text { - Uncontrolled case series of } 3 \text { severely ill patients } \\
\text { - Improvement in clinical status of all } 3 \text { patients }\end{array}$ & 17 \\
\hline SARS1 & Shenzhen, China & $\begin{array}{l}2 \text { units of } 250 \mathrm{~mL} \text { each } \\
\text { (total } 500 \mathrm{~mL} \text { ); } \\
\text { transfused } 12 \text { hours apart }\end{array}$ & Not stated & $\begin{array}{l}\text { - Letter to editor/case report of one patient } \\
\text { - Improvement in clinical status }\end{array}$ & 53 \\
\hline MERS & Seoul, South Korea & $250 \mathrm{~mL}$ & Not stated & $\begin{array}{l}\text { - Case report (letter to editor) of } 1 \text { patient } \\
\text { - Possible TRALI reported }\end{array}$ & 54 \\
\hline COVID-19 & Wuhan, China & $200 \mathrm{~mL}$ & $\begin{array}{l}\text { Neutralizing anti-SARS-CoV-2-antibody } \\
\text { titer }>1: 640\end{array}$ & $\begin{array}{l}\text { - Uncontrolled case series of } 10 \text { severely ill patients } \\
\text { - Other therapies included steroids, antimicrobials, antivirals } \\
\text { - Median onset of symptoms to CP } 16.5 \text { days (IQR } 11.0-19.3 \text { days) } \\
\text { - Improvement in clinical status of all patients } \\
\text { - No significant adverse effect }\end{array}$ & 22 \\
\hline
\end{tabular}

$\mathrm{CP}$, convalescent plasma; PRNT, plaque reduction neutralization assay; MN, microneutralization assay.

the presence of antibodies against SARS-CoV-2 and at least 14 days passing after the resolution of symptoms (e.g., fever, cough, shortness of breath).

Donor recruitment. Those who have recovered from COVID-19 will be recruited to serve as potential blood donors. Given the magnitude of the pandemic, finding donors is not anticipated to be a problem. Approaches include community outreach in areas with robust epidemics, advertising, and communication through media, and/or directly through providers (e.g., at time of discharge) and their professional organizations (e.g., databases, websites - http://ccpp19.org). There is also consideration about messaging those who receive positive results either prospectively or after the fact. The latter poses some ethical concerns, which weigh public health need against patient privacy and confidentiality. A limited waiver of HIPAA in the US may allow for greater freedom in this regard (26). Blood centers have well-developed infrastructure for donor recruitment; while they may be best equipped to oversee recruitment in collaboration with partner hospitals, their primary responsibility is to ensure an adequate blood supply to meet clinical demand. Confronted with recent, severe blood shortages given canceled blood drives, blood centers have been forced to prioritize their efforts accordingly, while still planning for convalescent plasma collection. The latter presents an additional burden on the blood centers, particularly while contending with the logistical constraints posed by COVID-19 (e.g., limited staffing, a contracted donor pool, travel restrictions, etc.). Of note, while convalescent plasma could compete with routine plasma collections, this may be offset by lowered demand for standard plasma, given COVID-19 mitigation measures, such as canceled elective surgeries.

Predonation screening to qualify convalescent donors. There is still uncertainty surrounding the optimal workflow for predonation screening. Heterogeneity in approaches based on local capacity and needs is expected. We have proposed a two-step process that divorces the blood center from the predonation screening; the predonation screening is left to the clinical provider, who performs an assessment of the donor, collects an NP swab for nucleic acid testing to confirm that the individual is virus free (i.e., in the event that a negative test has not yet been obtained), and collects a blood sample for antibody testing before referring the donor 


\section{Convalescent plasma collections workflow}

History of COVID-19

- Diagnosis of SARS-CoV-2 infection/COVID-19

- Positive test for SARS-CoV-2 using an EUA approved molecular assay or

- Evidence of antibodies against SARS-CoV-2

$\geq 14$ days following resolution of symptoms
* Suggest completion of donor history questionnaire during pre-donation screening to avoid unnecessary deferral for reasons that are unrelated to COVID-19 (e.g. risk of transfusion-transmissible infections)

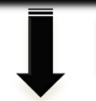

Pre-donation screening: Clinical provider*

- Clinical assessment of no sign of active infection (i.e. afebrile, no reported symptoms for $\geq 14$ days)

- Serum collection for anti-SARS-CoV-2

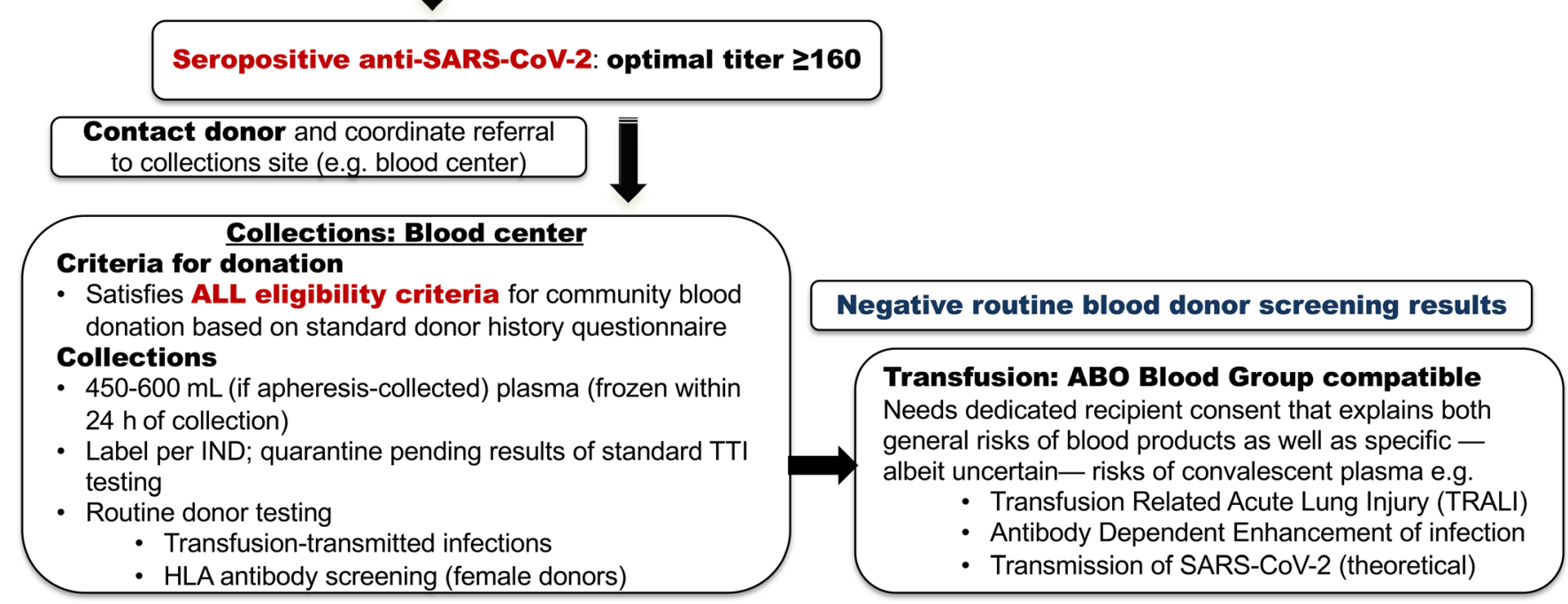

Figure 1. Convalescent plasma collections workflow. EUA, Emergency Use Authorization; TTI, transfusion-transmitted infection.

to a collection facility. Anti-SARS-CoV-2 provides evidence of resolved infection. Nonetheless, the original FDA guidance document mandated evidence of a negative molecular test to ensure a reasonable measure of caution. This recommendation reflects the overriding mandate to protect safety given the current state of knowledge, which associates the presence of SARS-CoV-2 RNA in NP specimens with potential infectivity. The eligibility criteria for donation of convalescent plasma have evolved, rapidly. For one, the presence of antibodies against SARS-CoV-2 is now permissible as evidence of prior COVID-19. For another, the requirement for a negative test (e.g., NP swab) in those 14 to 27 days following resolution of symptoms has since been relaxed.

Antibody testing. Antibody testing comes with its own challenges, as reflected in the FDA guidance document. In general, one cannot qualify donors or manufacture a therapeutic agent using tests that have not been vetted appropriately. However, there is uncertainty as to which antibodies are optimally effective in the context of COVID-19. Neutralizing antibodies are likely to correlate better with function. However, neutralizing antibody assays are not amenable to high-throughput screening in clinical laboratories and are not widely available. In contrast, quantitative assays (e.g., ELISA) are available, but commercially available assays have not been rigorously validated. Further, the relationship between total anti-SARS-CoV-2 antibodies and neutralizing anti-SARSCoV-2 antibodies remains unclear. There is also uncertainty as to whether total antibodies or subclasses (e.g., IgM, IgG, or IgA) are the optimal measure and which antigen is most informative; in this regard, various forms of the spike or S protein have been tested and used $(27,28)$.

Limited data are currently available on the ELISAs. One group reported findings demonstrating both "strong reactivity against $\operatorname{IgG3}$, IgM, and IgA" using assays targeting spike antigens and low crossreactivity when testing other human coronaviruses (27). Another group reported on performance of a point-of-care antibody test for combined detection of IgM and IgG, demonstrating a sensitivity and specificity of $88.7 \%$ and $90.6 \%$, respectively (29).

The antibody titer will be affected by the timing of collection relative to onset of infection. While data are limited, seroconversion has been observed to occur between 8 and 21 days after the onset of symptoms $(28,30)$. Coupled with reports from China of high titers of anti-SARS-CoV-2 antibodies in the overwhelming majority of convalescent patients, the findings suggest that units 
of plasma that are collected 14 days or more after resolution of symptoms should contain high titers of antibodies (22). In the setting of a temporizing therapy, one needs to balance acuity of need with a desire for a highly validated assay and a refined treatment modality. Indeed, the FDA guidance document manages this uncertainty by suggesting, rather than requiring testing, i.e., "defined SARS-CoV-2 neutralizing antibody titers, if testing can be conducted (e.g., optimally greater than or equal to 1:160)" (25). This will certainly change as antibody testing becomes more widely available. One could even foresee routine serosurveillance of blood donors, which would bypass the need for predonation screening, particularly if the convalescent plasma is produced from whole blood collections.

Collection and testing. Donors who have successfully completed predonation screening are directed to the blood center. We have developed a specialized form to alert the blood center of a convalescent plasma donor; the form confirms that all prescreening criteria have been met and that the plasma will be administered under IND. This ensures that donors have largely been vetted prior to collection. Upon presentation to the blood center, donors still need to qualify as community volunteer blood donors through completion of a donor history questionnaire and standard physical examination as specified by FDA and professional standards of practice. It is recommended that common deferrals be ruled out during predonation screening (e.g., through administration of the questionnaire) to minimize on-site deferral at the blood center for reasons that would otherwise disqualify the individuals from community donation (e.g., risk factors for transfusiontransmissible infections).

Apheresis (rather than whole blood donation) is recommended to optimize the yield of convalescent plasma. Apheresis refers to an automated technology in which whole blood is continuously centrifuged into its components (i.e., red blood cells, plasma, platelets); this allows for selective collection of the desired blood fraction with return of the other components to the donor. This is highly efficient: approximately 400 to $800 \mathrm{~mL}$ of plasma can be obtained from a single apheresis donation, which then provides 2 to 4 units of convalescent plasma for transfusion. The units are frozen within 24 hours of collection and quarantined - as is routine - pending results from standard blood donor testing. The latter fulfills regulatory requirements and mostly comprises testing for transfusion-transmissible infections (e.g., HIV, hepatitis B and $\mathrm{C}$ viruses, etc.). There is also required testing of female donors with a history of pregnancy for HLA antibodies to mitigate the risk of transfusion-related acute lung injury (TRALI).

Distribution of convalescent plasma. In the traditional model of blood collections in the US and other high-income countries, the blood center recruits its own voluntary nonrenumerated blood donors, after which there is equitable distribution based on need. The distribution model in COVID-19 employs convalescent individuals as "directed donors." The term directed donor typically refers to a friend or family member who donates specifically for a given patient. Directed donation is not actively encouraged, given that social pressure may disincentivize the donor's admission of high-risk behavior. In contrast, the COVID-19 model employs the process differently, directing units to institutions (i.e., hospitals) - rather than to individual patients - for transfusion to patients under emergency IND. Nonetheless, if institutions are left to recruit their own donors to support internal needs (i.e., for emergent use for individual patients), it raises the question of whether the blood centers have the ability to allocate units equitably. Many hospitals lack the experience and capacity to recruit donors, limiting their access to the supply of convalescent plasma. This model could also prove inefficient should donors pass predonation screening at the clinical provider, yet later fail qualification upon presentation to the blood center. Once adequate donors are recruited and high-throughput testing is available, the model will likely change.

Proposed under the FDA's expanded access program would be to regionalize or centralize recruitment, collections, and inventory management. Nonetheless, major obstacles remain with extant acuity of need and little time to construct an inventory as proposed.

Optimal dosing and transfusion. Historically, the dosing of convalescent plasma has been highly variable, which may be ascribed to differences by indication (i.e., prevention versus treatment). Pertinent to the current pandemic, a study in China employed a single $(200 \mathrm{~mL}$ ) unit of plasma (22). In the planned clinical trials, one unit has been proposed for use for postexposure prophylaxis and one to two units have been proposed for treatment. The antibodies' duration of efficacy is unknown, but is postulated to last weeks to a few months $(7,31)$. The selected dosing was based on experience with previous use of convalescent plasma therapy in SARS, where $5 \mathrm{~mL} / \mathrm{kg}$ of plasma at a titer of 1:160 or greater was utilized (16). Historically, prophylactic doses (in some cases only a quarter of that of the proposed treatment dose) have been used successfully. This was considered when designing the clinical trials. Considering first-order linear proportionality, $3.125 \mathrm{~mL} / \mathrm{kg}$ of plasma with a titer of greater than 1:64 would provide an equivalent immunoglobulin level to one-quarter of $5 \mathrm{~mL} / \mathrm{kg}$ plasma with a titer of 1:160 or greater.

For a typical patient $(\sim 80 \mathrm{~kg})$, this would result in $250 \mathrm{~mL}$ of plasma $(3.125 \mathrm{~mL} / \mathrm{kg} \times 80 \mathrm{~kg}=250 \mathrm{~mL}>1: 64)$, approximating the volume of a standard unit of plasma in the US. This scheme imparts logistical ease to product preparation for adult transfusions. In pediatric transfusions (trials are being planned), there will be the need to aliquot large volume units and dose by body weight. Given the current level of uncertainty, more precise models to estimate bioavailability in tissues where virus and host interact are not yet possible.

\section{Clinical trials to evaluate the safety and efficacy of human anti-SARS-CoV-2 plasma}

Despite a favorable historical record, few controlled trials have been performed to evaluate the efficacy of convalescent plasma, in large part due to its emergency application in times of epidemics. At least five clinical trials have been proposed to evaluate human anti-SARS-CoV-2 plasma for the prevention and treatment of COVID-19.

The first trial is of the use of human anti-SARS-CoV-2 plasma as post-exposure prophylaxis: a randomized, blinded phase 2 trial will be undertaken to compare the efficacy and safety of human anti-SARS-CoV-2 plasma versus control (SARS-CoV-2 nonimmune plasma) among adults (age $\geq 18$ years) who have had close 
contact exposure to COVID-19, but have not yet manifested symptoms. Per the US CDC, close contact exposure refers to being within approximately 6 feet ( 2 meters) of a patient with COVID-19 for a prolonged period of time (without personal protective equipment [PPE]). Close contact may occur while caring for, living with, visiting, or sharing a healthcare waiting area or room with a COVID-19 case or having direct contact with infectious secretions of a COVID-19-infected individual (e.g., being coughed on) without PPE. If found to be safe and effective, postexposure prophylaxis would offer an intervention for vulnerable populations (e.g., health care workers, immunocompromised patients, individuals with cardiovascular and respiratory disease, nursing home residents) following exposure. Prevention would confer direct clinical benefit for those at risk. Moreover, societal benefits would be wide ranging, including the protection of frontline workers in the COVID-19 pandemic.

The second trial will evaluate whether human anti-SARSCoV-2 plasma can help patients initially presenting with mild disease. The target population would comprise symptomatic individuals with confirmed SARS-CoV-2. The endpoints would be resolution of symptoms, prevention of hypoxemia on room air, or progression to severe disease, reflecting an interest in averting complications (and required hospitalization).

Third, the effect of human anti-SARS-CoV-2 plasma for moderately ill patients would be studied. The target population is hospitalized patients with COVID-19 who manifest symptoms - albeit not of sufficient acuity to warrant ICU admission (and specifically mechanical ventilation). Staving off progression to critical illness could avoid overburdening of critical care resources currently in shortage, such as mechanical ventilators.

A fourth trial will evaluate human anti-SARS-CoV-2 plasma treatment as a rescue intervention in patients who require mechanical ventilation due to COVID-19. This target group is important; however, it is also a group for which data are most difficult to interpret, given the likely presence of confounding variables, including other putative therapies for COVID-19.

Finally, a fifth trial will examine safety and pharmacokinetics convalescent plasma in high-risk pediatric patients. Children of all ages are susceptible to COVID-19 infection; while comparatively rare, severe disease and even deaths have been described in children (32), underscoring the need to address risk to children.

Complementing these five trials, studies are being designed to collect and mine data from emergency (i.e., compassionate) use of convalescent plasma or expanded access treatment.

\section{Potential risks}

Human plasma transfusion is a routine, daily event in modern hospitals. Human anti-SARS-CoV-2 plasma differs from standard plasma only by virtue of the presence of antibodies against SARSCoV-2. Donors will satisfy all criteria for blood donation based upon federal and state regulations for volunteer donor eligibility, and blood will be collected in FDA licensed blood centers.

Therefore, the risks to transfusion recipients are likely to be no different from those of standard plasma. Risk of transfusion-transmissible infection is very low in the US and other high-income countries. Typically cited estimates are less than one infection per two million donations for HIV, hepatitis B, and hepatitis C virus- es (33). There are also noninfectious hazards of transfusion, such as allergic transfusion reactions, transfusion-associated circulatory overload (TACO), and TRALI (34). While the risk of TRALI is generally less than one for every 5,000 transfused units, TRALI is of particular concern in severe COVID-19, given potential priming of the pulmonary endothelium. However, routine donor screening includes HLA antibody screening of female donors with a history of pregnancy to mitigate risk of TRALI (35). Of note, risk factors for TACO (e.g., cardiorespiratory disease, advanced age, renal impairment, etc.) are shared by those at risk of COVID-19, underscoring the need for careful attention to fluid volume management.

Specific risks pertaining to human anti-SARS-CoV-2 plasma include transfusion-transmitted SARS-CoV-2. This is largely theoretical, since the recipient is already infected and there has never been a report of transmission of a respiratory virus by blood transfusion. There is no donor screening in effect for common respiratory viruses, such as influenza, respiratory syncytial virus, and parainfluenza. SARS-CoV-2 is not considered to be a relevant transfusion-transmitted infection, and only $1 \%$ of symptomatic patients have been reported as having detectable SARS-CoV-2 RNA in their blood $(36,37)$. In Wuhan, 2430 blood donations were screened in real time (January 25 to March 4, 2020): a single $(0.04 \%)$ - asymptomatic - donor was found to be positive for SARS-CoV-2 RNA (38). A second (0.02\%) asymptomatic, SARSCoV-2 RNA-positive donor was identified on retrospective screening of 4995 donations (December 21 to January 22, 2020), and an additional two donors were identified as being RNA-emic through follow-up of donors who had developed fever subsequent to their donations. Nevertheless, donors will still need to wait 14 days following resolution of their symptoms to be eligible to donate.

There is also the theoretical possibility of antibody-dependent enhancement (ADE) following transfusion of human anti-SARSCoV-2 plasma. ADE refers to a process whereby antibodies that developed during a prior infection exacerbate clinical severity as a consequence of infection with a different viral serotype. This phenomenon is well known for some viruses, notably Dengue virus (39). The largely theoretical risk of ADE in COVID-19 would be attributable to antibodies potentiating infection upon exposure to other strains of coronavirus; this mechanism has been offered as a possible reason for the geographic variation in disease severity (40). Concerns about coronavirus-ADE stem primarily from in vitro studies using mAbs, whose relevance is uncertain to the polyclonal antibody composition found in convalescent plasma (41). In this regard, $\mathrm{mAbs}$ have been shown to have very different properties when acting as single molecules rather than in combination with other mAbs (42). Nonetheless, although ADE is unlikely to be relevant to the proposed use of convalescent plasma in prevention and treatment of a disease with the same virus, caution is warranted. Somewhat reassuring is the apparent absence of $\mathrm{ADE}$ reports with the use of convalescent plasma for SARS, MERS, or COVID-19.

For completion, it is unknown to what extent convalescent plasma might blunt the development of a natural immune response, especially when administered for prophylaxis.

\section{Risk benefit analysis}

In Figure 2, we constructed a stochastic age-specific susceptible-exposed-infected-removed (SEIR) model of COVID-19 

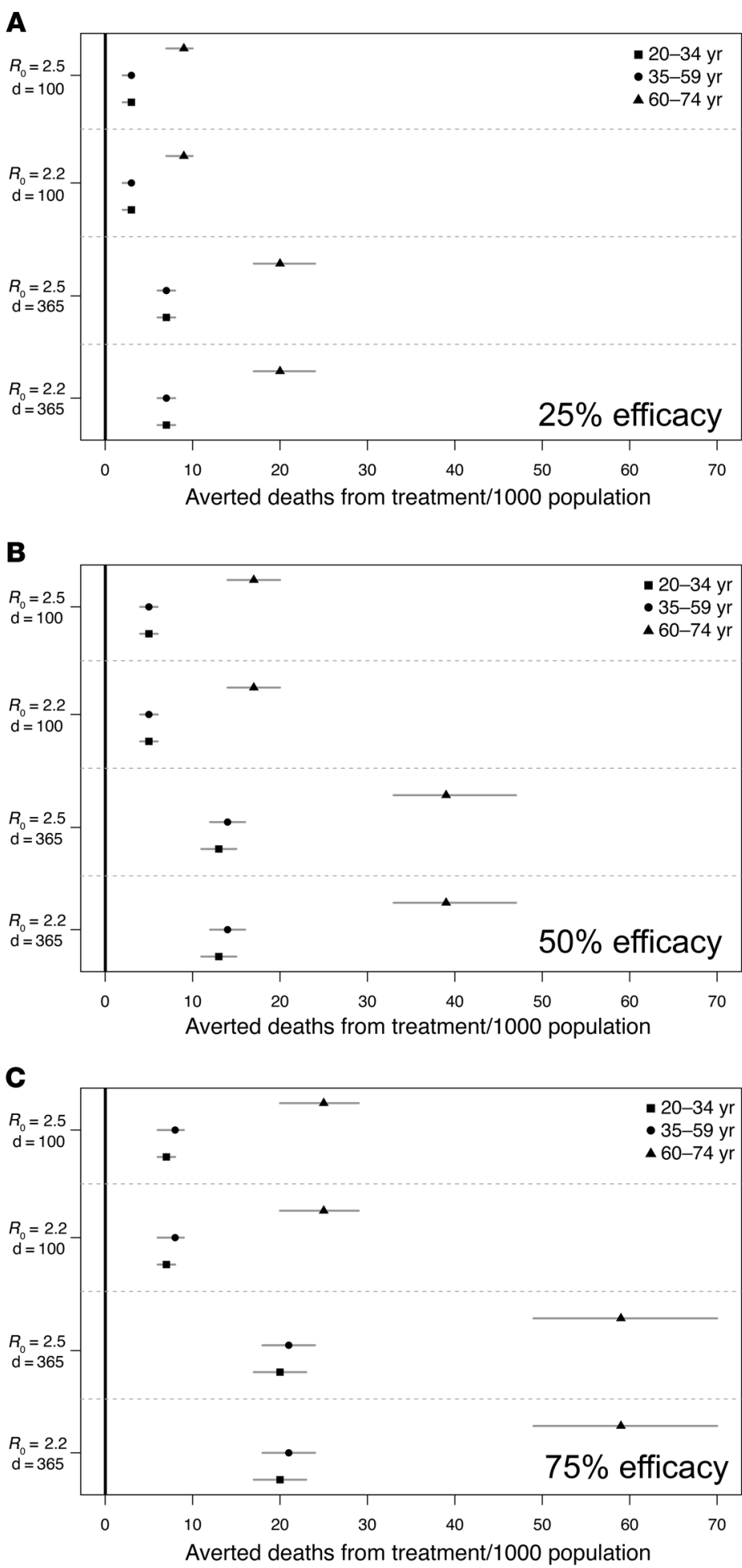

transmission reflective of the demography of Baltimore, Maryland, USA, to estimate the daily number of asymptomatic and symptomatic cases per day (43). Age-specific mixing was estimated using the POLYMOD data set for the United Kingdom obtained from the socialmxr $\mathrm{R}$ package (https://cran.r-project.org/ web/packages/socialmixr/socialmixr.pdf). The symptomaticto-asymptomatic ratio was set to $80 \% / 20 \%$ (44). Age-specific mortality rates were calculated using the age-specific case fatal-
Figure 2. Mortality risk with SARS-CoV-2 convalescent plasma versus control by age, reproductive number, projected efficacy of intervention, and time. Fatalities from SARS-CoV-2 were estimated by age groups assuming moderate and high $R_{0}$ scenarios over $100(d=100)$ or $365(d=365)$ days. We calculated the number of averted deaths from treatment per 1,000 individuals for a range of treatment efficacy: (A) $25 \%$, (B) $50 \%$, and (C) $75 \%$. We estimated these values using simulated incidence values from multiple runs of the transmission model. Results are shown for the mean (shown as a point) and $95 \%$ quantile. The estimated probability of death from a plasma transfusion was conservatively set at 41/3.6 million (solid black vertical line) (49).

ity ratio from the CDC (45). Age-specific severity rates were estimated using the National Center for Health Statistics on hypertension, diabetes, and cancer, where we assumed that the percentage of incident cases that become severe was roughly the same as the average percentage of individuals who have any of the above comorbidities. Transmission parameters were extracted from the literature to reflect both a moderate $\left(R_{0}=2.2\right)$ and high $\left(R_{0}=2.5\right)$ transmission setting (https://github.com/ midas-network/COVID-19/blob/master/parameter estimates/2019_novel_coronavirus/estimates.csv) (46). Multiple stochastic simulations were run $(n=500)$, with the $95 \%$ quantile and average provided. We considered incident cases for individuals between 20 and 74 to reflect the age range of healthcare workers. Healthcare workers may have a higher than population average contact rate with infected individuals; however, given uncertainties in this value, we adopted a conservative approach and assumed that mixing for healthcare workers was reflective of the general population without the deployment of any nonpharmaceutical interventions. Given uncertainties in the effectiveness of the intervention, we assumed $25 \%, 50 \%$, or $75 \%$ effectiveness. We then calculated the breakpoint where the fatality ratio would need to be higher than this value for the treatment to be worse than the fatality ratio from the disease.

The model highlights overwhelming benefit from prophylaxis or treatment with convalescent plasma even when conservative (e.g., 25\%) estimates of efficacy are modeled. For example, the proposed clinical trial was designed with a projected attack rate of $20 \%(10.5 \%-35 \%)(47,48)$; a high proportion of those who are infected will go on to severe disease, including death $(\sim 1 \%-4 \%)$ (2). In contrast, a total of 41 transfusion-associated fatalities ( 1 in 414,634 blood products) were reported to the FDA in 2015 (49). In short, blood transfusion in the US and other high-income countries is safe, and the associated risks are dwarfed by COVID-19associated morbidity and mortality.

\section{Conclusion}

The risks of COVID-19 infection are profound (50,51). Human plasma from recovered COVID-19 patients is projected to be a safe and potentially effective therapy for treatment and postexposure prophylaxis alike. Substantial evidence of benefit with 
prior use for viral infections offers strong precedent for such an approach. However, it is critically important to perform wellcontrolled clinical trials to confirm efficacy, thereby informing rational evidence-based decision making.

\section{Acknowledgments}

Any views or opinions that are expressed in this manuscript are those of the author's, based on his own scientific expertise and professional judgment; they do not necessarily represent the views of either the Blood Products Advisory Committee or the formal position of the FDA and also do not bind or otherwise obligate or commit either the Advisory Committee or the Agency to the views expressed. This manuscript was supported in part by a grant from the NIH (1K23HL151826) to EMB.

Address correspondence to: Evan M. Bloch, 600 North Wolfe Street/Carnegie 446 D1, Baltimore, Maryland 21287, USA. Phone: 410.614.4246; Email: ebloch2@jhmi.edu.
1. WHO. Rolling updates on coronavirus disease (COVID-19). http://www.who.int/emergencies/ diseases/novel-coronavirus-2019/events-asthey-happen. Updated April 3, 2020. Accessed April 7, 2020.

2. Center for Systems Science and Engineering at Johns Hopkins University. Coronavirus COVID19 Global Cases. http://coronavirus.jhu.edu/ map.html. Updated April 7, 2020. Accessed April 7, 2020.

3. Casadevall A, Pirofski LA. Antibody-mediated regulation of cellular immunity and the inflammatory response. Trends Immunol. 2003;24(9):474-478.

4. Casadevall A, Scharff MD. Serum therapy revisited: animal models of infection and development of passive antibody therapy. Antimicrob Agents Chemother. 1994;38(8):1695-1702.

5. Luke TC, Kilbane EM, Jackson JL, Hoffman SL. Meta-analysis: convalescent blood products for Spanish influenza pneumonia: a future $\mathrm{H} 5 \mathrm{~N} 1$ treatment? Ann Intern Med. 2006;145(8):599-609.

6. Casadevall A, Pirofski LA. The convalescent sera option for containing COVID-19. J Clin Invest. 2020;130(4):1545-1548.

7. Casadevall A, Scharff MD. Return to the past: the case for antibody-based therapies in infectious diseases. Clin Infect Dis. 1995;21(1):150-161.

8. Casadevall A, Dadachova E, Pirofski LA. Passive antibody therapy for infectious diseases. Nat Rev Microbiol. 2004;2(9):695-703.

9. Sahr F, et al. Evaluation of convalescent whole blood for treating Ebola virus disease in Freetown, Sierra Leone. J Infect. 2017;74(3):302-309.

10. Stokes J Jr, Wolman IJ, Carpenter HC, Margolis J. Prophylactic use of parents' whole blood in anterior Poliomyelitis: Philadelphia epidemic of 1932. Am J Dis Child. 1935;50(3):581-595.

11. Zhou B, Zhong N, Guan Y. Treatment with convalescent plasma for influenza A (H5N1) infection. N Engl J Med. 2007;357(14):1450-1451.

12. Hung IF, et al. Convalescent plasma treatment reduced mortality in patients with severe pandemic influenza A (H1N1) 2009 virus infection. Clin Infect Dis. 2011;52(4):447-456.

13. van Erp EA, Luytjes W, Ferwerda G, van Kasteren PB. Fc-mediated antibody effector functions during respiratory syncytial virus infection and disease. Front Immunol. 2019;10:548.

14. Gunn BM, et al. A role for Fc function in therapeutic monoclonal antibody-mediated protection against Ebola virus. Cell Host Microbe. 2018;24(2):221-233.e5.

15. Zhang JS, et al. A serological survey on neutralizing antibody titer of SARS convalescent sera.
JMed Virol. 2005;77(2):147-150.

16. Cheng Y, et al. Use of convalescent plasma therapy in SARS patients in Hong Kong. Eur J Clin Microbiol Infect Dis. 2005;24(1):44-46.

17. Yeh KM, et al. Experience of using convalescent plasma for severe acute respiratory syndrome among healthcare workers in a Taiwan hospital. JAntimicrob Chemother. 2005;56(5):919-922.

18. Ko JH, et al. Challenges of convalescent plasma infusion therapy in Middle East respiratory coronavirus infection: a single centre experience. Antivir Ther (Lond). 2018;23(7):617-622.

19. Arabi YM, et al. Feasibility of using convalescent plasma immunotherapy for MERS-CoV onfection, Saudi Arabia. Emerging Infect Dis. 2016;22(9):1554-1561.

20. huaxia. China puts 245 COVID-19 patients on convalescent plasma therapy. Xinhua. February 28, 2020. http://www.xinhuanet. com/english/2020-02/28/c_138828177.htm. Accessed April 7, 2020.

21. Shen C, et al. Treatment of 5 critically ill patients with COVID-19 with convalescent plasma [published online March 27, 2020]. JAMA. https://doi. org/10.1001/jama.2020.4783.

22. Duan K, et al. The feasibility of convalescent plasma therapy in severe COVID-19 patients: a pilot study. http://doi.org/10.1101/2020.03.16.2 0036145. Posted on medRxiv March 23, 2020.

23. Chen L, Xiong J, Bao L, Shi Y. Convalescent plasma as a potential therapy for COVID-19. Lancet Infect Dis. 2020;20(4):398-400.

24. Cao W, et al. High-dose intravenous immunoglobulin as a therapeutic option for deteriorating patients with coronavirus disease 2019. Open Forum Infect Dis. 2020;7(3):ofaa102.

25. Center for Biologics Evaluation and Research. Revised Information for Investigational COVID19 Convalescent Plasma. http://www.fda.gov/ vaccines-blood-biologics/investigationalnew-drug-ind-or-device-exemption-ideprocess-cber/investigational-covid-19convalescent-plasma-emergency-inds. Updated April 6, 2020. Accessed April 7, 2020.

26. COVID-19 \& HIPAA Bulletin. Limited Waiver of HIPAA Sanctions and Penalties During a Nationwide Public Health Emergency. U.S. Department of Health and Human Services; 2020.

27. Amanat F, et al. A serological assay to detect SARS-CoV-2 seroconversion in humans. http:// doi.org/10.1101/2020.03.17.20037713. Posted on medRxiv March 18, 2020.

28. Okba NMA, et al. SARS-CoV-2 specific antibody responses in COVID-19 patients. http://doi. org/10.1101/2020.03.18.20038059. Posted on
medRxiv March 20, 2020.

29. Li Z, et al. Development and clinical application of a rapid IgM-IgG combined antibody test for SARS-CoV-2 infection diagnosis [published online February 27, 2020]. J Med Virol. https:// doi.org/10.1002/jmv.25727.

30. Guo L, et al. Profiling early humoral response to diagnose novel coronavirus disease (COVID-19) [published online March 21, 2020]. Clin Infect Dis. https://doi.org/10.1093/cid/ciaa310.

31. Casadevall A. Passive antibody administration (immediate immunity) as a specific defense against biological weapons. Emerging Infect Dis. 2002;8(8):833-841.

32. Dong $Y$, et al. Epidemiological characteristics of 2143 pediatric patients with 2019 coronavirus disease in China [published online March 16, 2020]. Pediatrics. https://doi.org/10.1542/ peds.2020-0702.

33. Busch MP, Bloch EM, Kleinman S. Prevention of transfusion-transmitted infections. Blood. 2019;133(17):1854-1864.

34. Hendrickson JE, Hillyer CD. Noninfectious serious hazards of transfusion. Anesth Analg. 2009;108(3):759-769.

35. Standards Program Committee Blood Banks Transfusion Services Standards Committee. Standards for Blood Banks Transfusion Services, 31st ed. Bethesda, Maryland, USA: AABB; 2018.

36. FDA. Code of Federal Regulations. Title 21. Volume 7. Part 630. Donation suitability requirements. Section 630.30. Definitions. http://www. accessdata.fda.gov/scripts/cdrh/cfdocs/cfcfr/ CFRSearch.cfm?fr=630.3. Updated March 17, 2020. Accessed April 7, 2020.

37. Wang W, et al. Detection of SARS-CoV-2 in different types of clinical specimens [published online March 11, 2020]. JAMA. https://doi. org/10.1001/jama.2020.3786.

38. Chang L, Zhao L, Gong H, Wang L, Wang L. Severe acute respiratory syndrome coronavirus 2 RNA detected in blood donations [published online April 3, 2020]. Emerging Infect Dis. https:// doi.org/10.3201/eid2607.200839.

39. Katzelnick LC, et al. Antibody-dependent enhancement of severe dengue disease in humans. Science. 2017;358(6365):929-932.

40. Tetro JA. Is COVID-19 receiving ADE from other coronaviruses? Microbes Infect. 2020;22(2):72-73.

41. Wan Y, et al. Molecular mechanism for antibody-dependent enhancement of coronavirus entry. J Virol. 2020;94(5):e02015-19.

42. Chow SK, Smith C, MacCarthy T, Pohl MA, Bergman A, Casadevall A. Disease-enhancing 
antibodies improve the efficacy of bacterial toxin-neutralizing antibodies. Cell Host Microbe. 2013;13(4):417-428.

43. midas-network. COVID-19/parameter_estimates/2019_novel_coronavirus. http://github. com/midas-network/COVID-19/tree/master/ parameter_estimates/2019_novel_coronavirus. Accessed April 7, 2020.

44. Mizumoto K, Kagaya K, Zarebski A, Chowell G. Estimating the asymptomatic proportion of coronavirus disease 2019 (COVID-19) cases on board the Diamond Princess cruise ship, Yokohama, Japan, 2020. Euro Surveill. 2020;25(10):2000180.

45. CDC COVID-19 Response Team. Severe outcomes among patients with coronavirus disease 2019 (COVID-19) - United States, February 12-March 16, 2020. MMWR Morb Mortal Wkly Rep. 2020;69(12):343-346.
46. Kucharski AJ, et al. Early dynamics of transmission and control of COVID-19: a mathematical modelling study. Lancet Infect Dis. 2020;20(5):553-558.

47. Burke RM, et al. Active monitoring of persons exposed to patients with confirmed COVID-19 United States, January-February 2020. MMWR Morb Mortal Wkly Rep. 2020;69(9):245-246.

48. Liu Y, Eggo RM, Kucharski AJ. Secondary attack rate and superspreading events for SARS-CoV-2. Lancet. 2020;395(10227):e47.

49. Center for Biologics Evaluation and Research. Fatalities Reported to FDA Following Blood Collection and Transfusion Annual Summary for FY2017. Silver Spring, Maryland, USA: FDA; 201. http://www.fda.gov/media/124796/download. Accessed April 7, 2020.

50. Mizumoto K, Chowell G. Estimating risk for death from 2019 novel coronavirus disease,
China, January-February 2020 [published online March 13, 2020]. Emerging Infect Dis. https://doi. org/10.3201/eid2606.200233.

51. Yang X, et al. Clinical course and outcomes of critically ill patients with SARS-CoV-2 pneumonia in Wuhan, China: a single-centered, retrospective, observational study [published online February 24, 2020]. Lancet Respir Med. https:// doi.org/10.1016/s2213-2600(20)30079-5.

52. Wong VW, Dai D, Wu AK, Sung JJ. Treatment of severe acute respiratory syndrome with convalescent plasma. Hong Kong Med J. 2003;9(3):199-201.

53. Kong L. Severe acute respiratory syndrome (SARS). Transfus Apher Sci. 2003;29(1):101.

54. Chun S, et al. Possible transfusion-related acute lung injury following convalescent plasma transfusion in a patient with Middle East respiratory syndrome. Ann Lab Med. 2016;36(4):393-395. 